\title{
Controlling Complex Dynamical Networks
}

\author{
Guanrong Chen ${ }^{(\bowtie)}$ \\ City University of Hong Kong, Hong Kong, China \\ eegchen@cityu.edu.hk
}

In this talk, we discuss pinning control of complex dynamical networks, with emphasis on both state and structural controllabilities of networked higher-dimensional linear time-invariant dynamical systems coupled by directed and weighted higher-dimensional communication channels. We show how the network topology, node-system dynamics, external control inputs, and inner interactions affect the controllability of such a complex dynamical network. We present precise necessary and sufficient conditions for the network controllability in a general topology, as well as in some special configurations such as trees and cycles with more subtitle details. 